\title{
Characterization of $x$-ray imaging crystal spectrometer for high-resolution spatially-resolved x-ray Thomson scattering measurements in shock-compressed experiments
}

J. Lu, ${ }^{\text {a,b, }}{ }^{*}$ K. W. Hill ${ }^{c}$, M. Bitter ${ }^{c}$, N. A. Pablant ${ }^{c}$, L. F. Delgado-Aparicio ${ }^{c}$, P. C. Efthimion $^{\mathrm{c}}$, H. J. Lee ${ }^{\mathrm{d}}$, and U. Zastrau ${ }^{\mathrm{d}, \mathrm{e}}$

a Institute of nuclear physics and chemistry, Mianyang, Sichuan 621999, China

b Key Laboratory of Optoelectronic Technology and System of Ministry of Education, Chongqing University, Chongqing 400030, China

c Princeton Plasma Physics Laboratory, Princeton, NJ 08543, USA

d SLAC National Accelerator Laboratory, Menlo Park, CA 94025, USA

e Institut für Optik und Quantenelektronik (IOQ), Friedrich-Schiller-Universität Jena, Max-Wien

Platz 1, Jena 07743, Germany

* Corresponding author. Tel.: +86 $08162482749,+8615720564107$;

Email address: jianlu1003@Gmail.com

\begin{abstract}
We have proposed, designed and built a dual-channel x-ray imaging crystal spectrometer (XICS) for spectrally- and spatially-resolved x-ray Thomson scattering (XRTS) measurements in the Matter in Extreme Conditions (MEC) end station at Linac Coherent Light Source (LCLS). This spectrometer employs two spherically-bent germanium (Ge) 220 crystals, which are combined to form a large aperture dispersive element with a spectral bandwidth of $\sim 300 \mathrm{eV}$ that includes both the elastic and inelastic $\mathrm{x}$-ray scattering peaks. The apparatus and its characterization are described. A resolving power of $\sim 1900$ was demonstrated and a spatial resolution
\end{abstract}


of $\sim 12 \mu \mathrm{m}$ was achieved in calibration tests. For XRTS measurements, a narrow-bandwidth $(\Delta E / E<0.003)$ LCLS x-ray free electron laser (XFEL) beam at $5.07 \mathrm{keV}$ was used to probe a dense carbon plasma produced in shock-compressed samples of different forms of carbon. Preliminary results of the scattering experiments from Pyrolytic Graphite sample are presented that illustrate the utility of the instrument.

Key words: X-ray spectrometer, x-ray Thomson scattering, warm dense matter, high resolving power

\section{Introduction}

Warm dense matter (WDM) [1-2], with temperatures between 1 to $100 \mathrm{eV}$ and densities ranging from $10^{21}$ to $10^{24} \mathrm{~cm}^{-3}$, has lately become a subject of intense research, since it plays a crucial role in understanding of the convergence between condensed matter and plasma physics. Modern, state-of-the-art high energy density facilities [3-5] have made it possible to create warm dense matter in the laboratory. However, an accurate characterization of the physical parameters, such as temperature, density, and ionization state, etc., of WDM is experimentally challenging, since the temperatures are not high enough to excite $\mathrm{x}$-ray spectral emissions and since the high densities of free electrons make WDM opaque for visible light such that optical probes can not be used. X-ray Thomson Scattering (XRTS) [6-11], using an intense quasi-monochromatic $\mathrm{x}$-ray source which is typically provided by either laser-produced plasma (LPP) or an XFEL [12-13] for probing, is however a powerful 
diagnostic for WDM. Incident $\mathrm{x}$-rays with 3-10 keV or even higher [14] photon energies are directed into a sample, and spectrally- and spatially-resolved scattered $\mathrm{x}$-rays are observed in forward or backward scattering geometry using an x-ray spectrometer.

$\mathrm{X}$-ray scattering signals are weak due to a small scattering cross section, such that spectrometers with high collection efficiencies are required to obtain high quality data. Highly Oriented Pyrolytic Graphite (HOPG) [15-16] crystals in the von Hamos [17] configuration are a common choice for XRTS measurements as they offer superior brightness with the highest known integrated reflectivity in the mosaic-focusing mode [18] (magnification of 1), which enabled the proof-of-principle measurements [8]. Although with a very high reflectivity, its low spectral resolution $(E / \Delta E \sim 300)$ and poor spatial resolution of order of few millimeters, which results from the broad width of the crystal's rocking curve, is a significant disadvantage, especially that spatial resolution is an essential requirement for XRTS measurement to determine whether the scattering arises from a homogeneous or an inhomogeneous WDM state. Recently, Highly Annealed Pyrolytic Graphite (HAPG) [19] was introduced as a spectrometer crystal with considerably lower mosaicity. While the spectral resolution could be improved to $E / \Delta E \sim 1000$, the issue of poor spatial resolution remains.

An imaging x-ray crystal spectrometer for XRTS measurement using a toroidally-bent crystal was recently proposed by Gamboa et al. [20-21], who demonstrated that such an imaging spectrometer can provide high spatial resolution 
$(<25 \mu \mathrm{m})$ within a large field of view $(\sim 3 \mathrm{~mm})$ and spectral resolution $(4 \mathrm{eV})$, which is approximately twice better than that of a HAPG spectrometer. However, such a toroidally-bent crystal spectrometer must be aligned over six axes. And a defocusing due to subtle errors in source positioning and Bragg angle setting can degrade the spatial resolution significantly. In this work we avoid this type of drawback by introducing a spherically-bent crystal [22-24], since the alignment of the spectrometer would then be much simplified by the high degree of symmetry of a spherically-bent crystal. A spherically-bent crystal can also provide the high spectral and spatial resolutions, which are needed for XRTS experiments; and, moreover, it allows to produce a spatially-resolved image with adjustable magnifications.

In this paper, we demonstrate the capability of a two-channel spherically-bent crystal spectrometer, where the crystals are used in a von Hamos-like configuration to measure spectrally- and spatially-resolved XRTS signals from a shock-compressed WDM sample. Tailored laser shocks are driven into carbon targets to produce high compression. An LCLS XFEL beam with energy of $5.07 \mathrm{keV}$ and bandwidth of $15 \mathrm{eV}$ is used as a probe to penetrate into the dense carbon and measurements of the scattered $\mathrm{x}$-rays in the non-collective regime were performed in the backward scattering geometry.

\section{Spectrometer design}

The x-ray imaging crystal spectrometer makes use of spherically-bent crystals to analyze the energy of the scattered x-ray radiation by Bragg diffraction and to obtain 
spatially resolved images of the x-ray scattering WDM sample by utilizing the focusing properties of spherical mirrors. After Bragg diffraction by the crystal lattice, X-rays with different wavelengths are dispersed with different Bragg angles in the meridional plane, according to the Bragg condition:

$$
n \lambda=2 d \sin \theta
$$

where $n, \lambda, d$, and $\theta$ are the order of diffraction, the x-ray wavelength, the interplanar spacing of the Bragg reflecting crystal lattice planes, and the Bragg angle, respectively.

The focusing properties of a spherically-bent crystal result from the rotational symmetry of the configuration about the axis, which connects a point source to the center of the curvature of the crystal, i. e. the center of the crystal sphere. Hence, this configuration has some similarities to the von-Hamos configuration, with the essential difference being that the cylindrically-bent crystal is replaced by a spherically-bent crystal. The spherical symmetry of the crystal makes the imaging scheme less sensitive to misalignment and also makes it possible to choose almost arbitrary magnifications $M$, whereas the magnification $M=1$ for mosaic focusing. X-rays of a certain wavelength that are emitted from a point source are Bragg reflected from the crystal with the same Bragg angle and focused onto a point on the above-mentioned axis of symmetry, producing a monochromatic point-to-point image in the sagittal plane, which is perpendicular to the meridional plane [25-28]. The distances, $p$ and $q$, of the source to the crystal and the detector to the crystal are related by

$$
1 / p+1 / q=1 / f_{s}
$$


where $f_{s}=R /(2 \sin \theta)$ is the sagittal focal length, which is determined by the radius of curvature $R$ of the crystal and the Bragg angle $\theta$. Since a spherically-bent crystal has different focal lengths in the meridional and sagittal planes, the magnifications in the two directions are different when $\theta \neq 90^{\circ}$, meaning that in this case spatial focusing can only be achieved in one direction, while the radiation is defocused in the other direction. The crystal-detector distance is the variable used to achieve focusing. Ideally, in order to maintain focusing in the sagittal direction across the detector, for each wavelength, the detector must be aligned as shown in Fig. 1 (a). The surface of the detector must lie along the line in the meridional plane that connects the source and the center of the curvature of the crystal. However, ray tracing calculations show that a detector length of $76 \mathrm{~mm}$ would be required for our application, which could be implemented by using a film or image plate. To fit to an electronically read-out CCD detector, which is $27.65 \mathrm{~mm}$ wide, the detector was positioned perpendicular to the reflected rays from the crystals, as shown in Fig. 1(a). In this arrangement, sagittal focusing can only be achieved over a small region.

The small size of detection area of the CCD detector also restricts the spectra range that can be observed using a single crystal. One possibility to cover a larger spectral range is to use a combination of several spherically-bent crystals with the same or different radii of curvatures. Here, we present a design in which the dispersive element is a set of two crystals with different radii in a non-concentric geometry, as shown in Fig. 1 (a). The two crystals, C1 and C2, chosen for a lower energy band and a higher energy band, were both Ge 220 crystals, which were 
spherically bent to radii of curvature of $210 \mathrm{~mm}$ (C1) and $222.8 \mathrm{~mm}$ (C2), respectively, and were operated in a $\theta<45^{\circ}$ regime. The crystal bending is done with great care to avoid a fracturing of the crystal lattice and to obtain high spectral and spatial resolutions. In order to fit the spectrometer into the MEC chamber, the two crystals were viewing the source with an angular tilt of $\sim 27.84^{\circ}$ and $\sim 20.02^{\circ}$ with respect to the horizontal source plane; see in Fig. 1(a). Main parameters of XICS are listed in Table I. The source-to-crystal distances for C1 and C2 are $247.3 \mathrm{~mm}$ and $296.5 \mathrm{~mm}$ and the crystal-to-detector distances are $515.3 \mathrm{~mm}$ and $468 \mathrm{~mm}$, yielding spatial magnifications of 2.08 and 1.59 , respectively. The crystal size is $18 \mathrm{~mm} \times 15$ $\mathrm{mm}$ and the Bragg angles are $39.34^{\circ}$ for $\mathrm{C} 1$ and $37.75^{\circ}$ for $\mathrm{C} 2$.

Table I The parameters of the XICS

\begin{tabular}{ccc}
\hline Parameter & C1 & C2 \\
\hline Bragg angle $\theta\left(^{\circ}\right)$ & 39.34 & 37.75 \\
Tilting angle $\varphi\left(^{\circ}\right)$ & 27.84 & 20.02 \\
Source-Crystal distance $p(\mathrm{~mm})$ & 247.3 & 296.5 \\
Crystal-Detector distance $q(\mathrm{~mm})$ & 515.3 & 468.0 \\
Sagittal magnification $M_{\mathrm{s}}$ & 2.08 & 1.59 \\
Meridional magnification $M_{\mathrm{m}}$ & 3.67 & 2.30 \\
\hline
\end{tabular}

The spectra from the two crystals were imaged onto spatially-separated segments of a single, back illuminated, deep-depletion CCD detector (Princeton Instruments PI MTE 2048B) containing $2048 \times 2048$ pixels of $13.5 \mu \mathrm{m}$ pitch. The depth of the photosensitive epitaxial silicon layer is extended, increasing the probability for photo-absorption and resulting in higher quantum efficiency [29], which is approximately 0.47 at $5.07 \mathrm{keV}$. 
Fig. 1(b) shows the CAD model of the spectrometer. Both crystals were placed in a light-tight chamber made of aluminum plates, which was chosen for its light weight and lower scattering cross-section. A metal pointer mounted in front of the crystal chamber with certain length and angle, determined by laboratory calibration, was used to align the spectrometer inside the MEC target chamber relative to the target. A $7 \mu \mathrm{m}$ thick aluminum foil as well as two $50 \mu \mathrm{m}$ aluminized Mylar foils were used as filters to shield the detector from visible light.

\section{Resolving power and spatial resolution calibration}

To quantify and qualify the spectrometer performance with respect to its spectral and spatial resolution, calibration experiments were completed with sources of a micro-focus x-ray tube and by x-ray fluorescence inside the MEC target chamber at LCLS.

Over a wide range of energy, the dispersion $(\mathrm{d} \lambda / \mathrm{dx})$ of a spherically-bent crystal spectrometer varies with the x-ray energy [30]. However, it can be regarded as approximately constant in a narrow spectral window. Positioning the detector perpendicular to the reflected rays from the crystal also helps improve the linearity of the dispersion, which eases the analysis of the scattered spectra. Fig. 2(a) shows a spatial (vertical) -spectral (horizontal) image of the raw experimental data of tungsten spectra from calibration tests obtained by the spectrometer using a micro-focus tungsten x-ray tube and a Pilatus 100k hybrid pixel array detector [31] with $487 \times 195$ photon counting pixels of size $172 \times 172 \mu \mathrm{m}^{2}$. Obviously, the spectra are spatially 
focused over a small region and spread out at both ends. The corresponding intensity profiles are shown in Fig.3 (a) and (c). In this calibration, both crystals are operated in $2^{\text {nd }}$ order reflection.

The experimental data agree well with the linear fittings, in which the slopes of the fitted lines represent the linear dispersions, shown in Fig.3 (b) and (d). The linear dispersions calculated from the $\mathrm{W}$ spectra in $2^{\text {nd }}$ order were $1.57 \mathrm{eV} /$ pixel for $\mathrm{C} 1$ and $2.12 \mathrm{eV} /$ pixel for $\mathrm{C} 2$. Since the linear dispersion is proportional to the reflection order and the pixel pitch, when converted to the $\mathrm{CCD}$ detector of $13.5 \mu \mathrm{m}$ pitch, the corresponding values were $0.06161 \mathrm{eV} /$ pixel and $0.0832 \mathrm{eV} /$ pixel, respectively.

For the calibration inside the MEC target chamber, we tuned the LCLS XFEL beam energy to $5.53 \mathrm{keV}$, which is above the K-edge of Vanadium, $5.456 \mathrm{keV}$. A 100 $\mu \mathrm{m}$ thick Vanadium foil was excited by this XFEL beam with a spot size of $15 \mu \mathrm{m}$ to produce $\mathrm{V} \mathrm{K} \alpha_{1}$ at $4952.2 \mathrm{eV}$ and $\mathrm{K} \alpha 2$ at $4944.6 \mathrm{eV}$ emission lines. The titanium $\mathrm{K} \beta_{1}$ line at $4931.8 \mathrm{eV}$ was also observed by irradiating a $\mathrm{Ti}$ foil. Both spectra were recorded by only the $\mathrm{C} 1$ spectrometer and the raw data was shown in Fig 2(b). The linear dispersion derived from those three lines was $0.06123 \mathrm{eV} /$ pixel, which differs by only $0.6 \%$ from the value of 0.06161 derived from the $\mathrm{W}$ data, as shown in Fig 3(e) and (f). With such a high accuracy, it is reasonable to believe that the dispersion of $\mathrm{C} 2$ derived from $\mathrm{W}$ spectra can also be used for calibration even though we lacked emission lines in MEC station for this crystal. The spectra range of $\mathrm{C} 1$ was calculated from the V spectra, which was from $4839.7 \mathrm{eV}$ to $4965.1 \mathrm{eV}$. For C2, we scattered the LCLS beam at $5.07 \mathrm{keV}$ from a cold carbon sample and recorded the elastic peak. 
From this peak position and the dispersion discussed above, we inferred that crystal 2 covered a spectral range from $4979.3 \mathrm{eV}$ to $5149.6 \mathrm{eV}$. Hence the spectrometer covered a spectral range of approximately $\sim 300 \mathrm{eV}$. Unfortunately, there is an energy gap of $14.2 \mathrm{eV}$ between two crystals. This energy gap can be eliminated by more careful calibration at the cost of narrowing the spectral range.

The measured line widths of this work were determined by Lorentzian fits to the data, which is made up of several broadening contributions, including the natural line width, the source broadening, the instrumental broadening and the broadening due to the crystal rocking curve width. The full width of half maximum (FWHM) of the measured $\mathrm{W} \mathrm{L} \beta_{1}$ and $\mathrm{L} \beta_{2}$ lines are a convolution of those four parts. For $\mathrm{W}$ lines, since the source size of the tungsten tube was only $8.5 \mu \mathrm{m}$, as measured by knife-edge technique [32], the source-size broadening was approximately $\sim 0.13 \mathrm{eV}$. The broadening due to the crystal rocking curve width can be simply estimated from $\Delta E / E$ $=(\Delta \theta / \tan \theta)$, yielding a $\Delta E \sim 0.2 \mathrm{eV}$ for both $\mathrm{L} \beta_{1}$ and $\mathrm{L} \beta_{2}$ lines. For Ti and $\mathrm{V}$ lines, using XOP XCRYSTAL_BENT routine [33], we calculated the reflectivity of perfectly bent Ge 220 crystal at $4952.2 \mathrm{eV}$ with a $\Delta \theta$ of $75 \mathrm{mrad}$, gives a rocking curve broadening of $0.55 \mathrm{eV}$. Source broadening is estimated to be $0.12 \mathrm{eV}$ with source size of $15 \mu \mathrm{m}$. After deconvolution with the source-size and rocking-curve broadenings, the actually measured line widths with XICS were achieved. A comparison of the measured widths of W, Ti and V lines from XICS with the values published by Salem and Lee [34], AXAF Project Science [35] and Gokhale et al. [36] are listed in table II. The measured $\mathrm{W} \mathrm{L} \beta_{1}$ line width in this work agrees well with Gokhale's value. The best 
spectral resolving power is 1912 at $\mathrm{V} \mathrm{K} \alpha_{2}$ line.

Table II Comparison of the measured W, Ti and V line widths with values published by Ref. [34],

\begin{tabular}{ccccccc}
\hline \multicolumn{7}{c}{$[35]$ and [36]. } \\
\hline Lines & $\begin{array}{c}\text { Energy } \\
(\mathrm{eV})\end{array}$ & $\begin{array}{c}\text { Salem and Lee } \\
(\mathrm{eV})\end{array}$ & $\begin{array}{c}\text { AXAF Proj. Sci. } \\
(\mathrm{eV})\end{array}$ & $\begin{array}{c}\text { Gokhale } \\
(\mathrm{eV})\end{array}$ & $\begin{array}{c}\text { Present work } \\
(\mathrm{eV})\end{array}$ & $E / \Delta E$ \\
\hline $\mathrm{W} \mathrm{L} \beta_{1}$ & 9672.3 & 6.9 & $7.33 \pm 0.3$ & $7.33 \pm 0.3$ & 7.28 & 1328 \\
$\mathrm{~W} \mathrm{~L} \beta_{2}$ & 9961.5 & 9.06 & $9.26 \pm 0.93$ & $/$ & 10.76 & 925 \\
$\mathrm{Ti} \mathrm{K} \beta_{1}$ & 4931.8 & $/$ & 1.96 & $/$ & 2.96 & 1666 \\
$\mathrm{~V} \mathrm{~K} \alpha_{1}$ & 4944.6 & $/$ & $/$ & $/$ & 3.03 & 1631 \\
$\mathrm{~V} \mathrm{~K} \alpha_{2}$ & 4952.5 & $/$ & $/$ & $/$ & 2.59 & 1912 \\
\hline
\end{tabular}

In order to evaluate the achievable spatial resolution, the LCLS x-ray beam was used to irradiate a $10 \mu \mathrm{m}$ thick titanium foil, producing $\mathrm{Ti} \mathrm{K} \beta_{1}$ line. The beam spot size was focused down to $15 \mu \mathrm{m}$ in diameter on the Ti foil. The spatial resolution is limited by the detector pitch and spatial magnification. To obtain a resolution better than $10 \mu \mathrm{m}$ would require a magnification at least of 2 for a detector pixel size of 13.5 $\mu \mathrm{m}$ with effective pixel size closer to $18-20 \mu \mathrm{m}$ due to charge sharing between neighboring pixels, which is on the order of a few $\mu \mathrm{m}$ [29]. Both the raw data of a well-focused $\mathrm{Ti} \mathrm{L} \beta_{1}$ line and spectrally-integrated, spatial (vertical) profile of the best focused region of the line are shown by dots in Fig 4(a) and (b) respectively. A Gaussian fit gives a FWHM of $\sim 40 \mu \mathrm{m}$. Assuming an x-ray source size of $15 \mu \mathrm{m}$, one infers an instrumental spatial resolution of $\sim 12 \mu \mathrm{m}$. If considering the charge sharing which was approximately $4.5 \mu \mathrm{m}$ [29], the spatial resolution would up to be $11.2 \mu \mathrm{m}$. The $15 \mu \mathrm{m}$ source was imaged on the detector with the size of $18.7 \mu \mathrm{m}$. This value is bigger than the true source size, which might be caused by defocusing due to positioning error of the crystal and detector. 


\section{Shock-compressed Thomson scattering measurements}

Thomson scattering is the scattering of electromagnetic radiation by free, weaklyand tightly-bound electrons. High energy x-rays are required for probing dense matter. In previous XRTS experiments for characterization of WDM, typically energetic lasers were employed to irradiate foil targets producing spectrally narrow K-shell emissions from helium-like and hydrogen-like atoms as a probe. One of the disadvantages of such sources is that the proximity of the intercombination line and dielectronic satellites result in an effective $\operatorname{He} \alpha[8,37]$ or $\operatorname{Ly} \alpha$ [39] source width of $\Delta E / E \sim 10^{-2}$. Moreover, those lines in the source spectrum may blend with down scattered plasmons and Compton peaks and distort the shape. Especially in the collective regime [39], small energy shifts requires a narrow x-ray probe bandwidth of order of $\Delta E / E \sim 0.002$ to fully resolve the shifted plasmon peak and unshifted inelastic scattering feature. To overcome the problem of source bandwidth we pursued the use of an X-ray free electron laser beam as a probe. The Linac Coherent Light Source [40] at SLAC is an x-ray free electron laser, tunable in the range 800 to $8000 \mathrm{eV}$ with $2 \times 10^{12}$ photons in a $20-200$ femtosecond $(f \mathrm{~s})$ pulse, which is a suitable facility for this application. In this study, the LCLS beam provides the narrow $(\Delta E / E<0.003)$ line with a bandwidth of $\sim 15 \mathrm{eV}$ at $5.07 \mathrm{keV}$ required for spectrally resolved x-ray scattering, which is desirable for scattering in both non-collective and collective regimes. Additionally, the LCLS beam provides the same number of x-ray photons compared with LPP but with much shorter pulse duration which could also enable high repetition measurements with higher temporal resolution of order of $20-200 \mathrm{fs}$ 
Fig. 5 shows a schematic of the experiment performed in the MEC instruments at LCLS. The material probed here was $100 \mu \mathrm{m}$ thick Pyrolytic Graphite (PG) at an initial density of $2.2 \mathrm{~g} / \mathrm{cc}$. Other carbon containing targets were also studied, but here we reported on only the PG data. Two MEC long pulse laser beams, focused down to $50 \mu \mathrm{m}$ diameter, irradiated the PG sample from both sides with approximately $9 \mathrm{~J}$ of $527 \mathrm{~nm}$ laser light in a $5 \mathrm{~ns}$ square pulse at an intensity of $\sim 4 \times 10^{13} \mathrm{~W} / \mathrm{cm}^{2}$, launching planar shocks. In addition to the heater beams, after a delay of several nanoseconds, an LCLS XFEL beam at $5.07 \mathrm{keV}$, focused down to $15 \mu \mathrm{m}$ diameter was used to penetrate into the $\mathrm{C}$ plasma to probe the compressed plasma.

The $5.07 \mathrm{keV}$ x-rays were scattered and partially absorbed by the high density $\mathrm{C}$ plasma. We observed the scattered radiation in near backscattering geometry $\left(\theta \approx 125^{\circ}\right)$ to maximize observable frequency shifts and to minimize effects of $k$-vector smearing due to finite probe and collection solid angle. Here $k$, the scattered wave number, is defined as the difference between the wave number of incident and the scattered $\mathrm{x}$ rays [8]. The scattering regime is characterized by the scattering parameter $\alpha$, which is a function of scattering vector $k$ and Debye screening length $\lambda_{\mathrm{D}} \cdot k=\left(4 \pi E_{0} / h c\right) \sin \left(\theta_{\mathrm{s}} / 2\right)$ and can be set by choosing the energy of the probe x-rays $E_{0}$, and the angle of the detected scattered x-rays $\theta_{\mathrm{s}}$. And $\lambda_{\mathrm{D}}=7.43 \times 10^{3}\left(T_{e} / n_{e}\right)^{-1 / 2}$, is a relation between electron temperature $T_{e}$ and density $n_{e}$. For $E_{0}=5.07 \mathrm{keV}$ and $\theta \approx 125^{\circ}, k$ is estimated to be 4.56 $\AA^{-1}$. For $T_{e}=4 \pm 1 \mathrm{eV}$ and $n_{e}=2 \times 10^{23} \mathrm{~cm}^{-3}, \lambda_{D}$ is approximately $0.33 \AA$. Therefore, the scattering parameter $\alpha=0.66<1$, indicating that the non-collective behavior of the 
plasma was probed in this experiment. In the non-collective regime, scattering from individual free electrons results in an unshifted elastic peak and a Compton peak, downshifted in the energy, that provide information on the electron velocity distribution through Doppler broadening. If the plasma is non-degenerate, probing the velocity distribution provides a measure of the electron temperature. The ratio of the downshifted Compton peak to the unshifted elastic peak gives an indication of the ionization state of the plasma and electron density.

Figure 6(a) gives the raw scattering spectra. As the sagittal magnification is changing with detector position, corrections were applied to the measured spectra [27]. As the spectral dispersion was calculated to be constant in Section III, no additional geometrical correction was needed. Sagittal magnification correction was used to straighten out the spectra while properly redistributing the intensity per unit area in the data. A corrected cross-section profile with background removed is shown in Fig 6(b). Two main features include the elastic and inelastic Compton feature at $\sim 5070 \mathrm{eV}$ and $\sim 990 \mathrm{eV}$, respectively. The Compton feature is down-shifted by the Compton energy, determined by $E_{\mathrm{c}}=(h k / 2 \pi)^{2} /\left(2 m_{e}\right)$, where $k$ is about $4.56 \AA^{-1}$ in our case, and the spectrum was broadened by the electron velocity distribution due to Doppler shifting. Crystal 2 recorded the elastic peak and part of the Compton peak and crystal 1 recorded the remainder of the Compton peak, shown in the inserted image in Fig. 5.

In Fig. 7, the spatial profiles of the elastic peak from both driven and undriven shots are compared. The spatial widths of the heated spectra are narrower than that of the cold spectra, suggesting that the spatial compressions were successfully observed 
and resolved. The LCLS beams probed the plasmas after a 5 ns delay for both measurements. Previously, few shots with strong, single-sided shocks were conducted and the spatial profiles were measured. It showed an obvious compression on the driven side, while the cold side remained unchanged. Consequently, one can conclude that the driven shocks should be responsible for spatial width compression. More interestingly, from Fig. 7, driving the sample with the same energy lasers from both sides shows a relatively symmetric compression, as compared to using different energies. These data also directly demonstrate the high spatial resolution capability of the crystal spectrometer. Note that there were two peaks appearing in "Undriven" profile for both shots. The same features were also shown simultaneously in the "Driven" profiles. We assumed that it might be caused by the spectrometer. However, this is not yet well understood.

\section{Conclusions}

In summary, we have developed and fielded an x-ray imaging crystal spectrometer with spherically-bent crystals in MEC target chamber, showing the capability of measuring XRTS radiation from the shocked warm dense matter sample with high spectral and spatial resolution. Characterizations of the spectrometer were performed with $\mathrm{W}$ L-series, $\mathrm{Ti} \mathrm{K}$ lines and $\mathrm{V}$ line for a dual-channel crystal spectrometer, demonstrating resolving power higher than 1900 and spatial resolution of order of $12 \mu \mathrm{m}$. Experimental data of XRTS measurements using an XFEL beam for probing shock-compressed carbon plasmas were also provided. Comparison shows that compression due to laser shocks was spatially resolved. 
For future applications, we plan to explore using imaging geometries with larger magnification in order to achieve better spatial resolution higher than $10 \mu \mathrm{m}$. However, for a given detector size, larger magnification can be achieved but at the expense of reduced spectral range. To improve the signal to noise ratio for obtaining benchmark quality data, higher throughput can be achieved using larger crystals, $60 \mathrm{~mm}$ rather than the present $15 \mathrm{~mm}$, and using thinner protective foils with $90 \%$ transmission, as compared to the present $48 \%$ transmission.

\section{Acknowledgements}

We wish to thank Peter Sloboda in PPPL and Brice Arnold in SLAC for help in the mechanical designing and fabrication of the spectrometer.

This work was performed under the auspices of the (U.S.) Department of Energy (DOE) by Princeton Plasma Physics Laboratory (PPPL) under Contract No. DE-AC02-09CH-11466. Ulf Zastrau in SLAC is a Peter-Paul-Ewald-Fellow of the Vokswagen Foundation.

\section{References}

[1] R. W. Lee, H. A. Baldis, R. C. Cauble, O. L. Landen, J. .S Wark, A. Ng, S. J. Rose, C. Lewis, D. Riley, J. C. Gauthier and P. Audebert, Laser. Part. Beams 20: 527(2002).

[2] S. Ichimaru, Rev. Mod. Phys. 54: 1017(1982).

[3] E. I. Moses, R. N. Boyd, B. A. Remington, C. J. Keane and R. Al-Ayat, Phys. Plasmas 16: 041006 (2009). 
[4] T. R. Boehly, D. L. Brown, R. S. Craxton, R. L Keck, J. P. Knauer, J. H. Kelly, T. J. Kessler, S. A. Kumpan, S. J. Loucks, S. A. Letzring, F. J. Marshall, R. L. McCrory, S. F. B. Morse, W. Seka, J. M. Soures and C. P. Verdon, Opt. Commun., 133: 495-506(1997).

[5] J. E. Bailey, T. Ao, E. Harding, S. B. Hansen, M. P. Desjarlais, R. W. Lemke, G. A. Rochau, J. Reneker and D. Romero, Sandia National Laboratories Report SAND 2012-7998(2012).

[6] O. L. Landen, S. H. Glenzer, M. J. Edwardsm, R. W. Lee, G. W. Collins, R. C. Cauble, W. W. Hsing and B. A. Hammel, J. Quant. Spectrosc. Radiat. Transfer 71: 465-478 (2001).

[7] G. Gregori, S. H. Glenzer, W. Rozmus, R. W. Lee and O. L. Landen, Phys. Rev. E 67: 026412(2003).

[8] S. H. Glenzer, G. Gregori, R. W. Lee, F. J. Rogers, S. W. Pollaine, and O. L. Landen, Phys. Rev. Lett. 90: 175002(2003).

[9] S. H. Glenzer and R. Redmer, Rev. Mod. Phys. 81: 1625(2009).

[10] H. J. Lee, P. Neumayer, J. Castor, T. Döppner, R. W. Falcone, C. Fortmann, B. A. Hammel, A. L. Kritcher, O. L. Landen, R. W. Lee, D. D. Meyerhofer, D. H. Munro, R. Redmer, S. P. Regan, S. Weber, and S. H. Glenzer, Phys. Rev. Lett. 102:115001(2009).

[11] A. L. Kritcher, P. Neumayer, J. Castor, T. Döppner, R. W. Falcone, O. L. Landen, H. J. Lee, R. W. Lee, E. C. Morse, A. Ng, S. Pollaine, D. Price, S. H. Glenzer, Science 322 (5898): 69-71 (2008). 
[12] R. W. Lee, S. J. Moon, H. K. Chung, W. Rozmus, H. A. Baldis, G. Gregori, R. C. Cauble, O. L. Landen, J. S. Wark, A. Ng, S. J. Rose, C. L. Lewis, D. Riley, J. C. Gauthier and P. Audebert, J. Opt. Soc. Am. B, 20: 770-778(2003).

[13] S. P. Hau-Riege, A. Graf, T. Döppner, R. A. London, J. Krzywinski, C. Fortmann, S. H. Glenzer, M. Frank, K. Sokolowski-Tinten, M. Messerschmidt, C. Bostedt, S. Schorb, J. A. Bradley, A. Lutman, D. Rolles, A. Rudenko and B. Rudek, Phys. Rev. Lett. 108: 217402 (2012).

[14] T. Ma, T. Döppner, R. W. Falcone, L. Fletcher, C. Fortmann, D. O. Gericke, O. L. Landen, H. J. Lee, A. Pak, J. Vorberger, K. Wünsch and S. H. Glenzer, Phys. Rev. Lett. 110: 065001(2013).

[15] U. Zastrau, C. R. D. Brown, T. Döppner, S. H. Glenzer, G. Gregori, H. J. Lee, H. Marschner, S Toleikis, O Wehrhan and E Förster, J. Instrum. 7: P09015(2012).

[16] H. Legall, H. Stiel, V. Arkadiev and A. A. Bjeoumikhov, Opt. Express, 14(10): 4570-4576 (2006).

[17] L. Von Hamos, Ann. Phys. 17: 716(1933).

[18] G. E Ice and C. J. Sparks, Nucl. Instrum. Methods Phys. Res. A, 291: 110-116(1990).

[19] U. Zastrau, A. Woldegeorgis, E. Forster, R. Loetzsch, H. Marschnera and I. Uschmann, J. Inst. 8:P10006(2013).

[20] E. J. Gamboa, D. S. Montgomery, I. M. Hall and R. P. Drake, J. Instrum., 6: P04004(2011).

[21] E. J. Gamboa, C. M. Huntington, M. R. Trantham, P. A. Keiter, R. P. Drake1, 
D. S. Montgomery, J. F. Benage and S. A. Letzring, Rev. Sci. Instrum. 83, 10E108 (2012).

[22] T. Ao, E. C. Harding, J. E. Bailey, et al., J. Quant. Spectrosc. Radiat. Transf. 144; 92-107 (2014).

[23] E. C. Harding, T. Ao, J. E. Bailey, et al., Rev. Sci. Instrum. 86: 043504 (2015).

[24] T. Ao, E. C. Harding, J. E. Bailey, et al., High Energy Density Physics, 18: 26 (2016).

[25] T. A. Pikuz, A. Ya. Faenov, S. A. Pikuz, et al., J. X-ray Sci. Technology, 5: 323 (1995).

[26] B. K. F. Young, A. L. Osterheld, D. F. Price, et al., Rev. Sci. Instrum. 60: 4049 (1998).

[27] D. B. Sinars, G. A. Chandler, J. E. Bailey, et al., J. Quant. Spectrosc. Radiat. Transf. 99: 595 (2006).

[28] G. N. Hall, S. A. Pikuz, T. A. Shelkovenko, et al., Phys. Plasmas 13: 082701 (2006).

[29] F.Zamponi, T. Kämpfer, A. Morak, I. Uschmann, and E.Förster, Rev. Sci. Instrum. 76: 116101(2005).

[30] S. A.Pikuz, J. D Douglass, T. A. Shelkovenko, D. B. Sinars and D.A. Hammer, Rev Sci Instrum. 79(1): 013106(2008).

[31] Ch. Broennimann, E. F. Eikenberry, B. Henrich, R. Horisberger, G. Huelsen, E. Pohl, B. Schmitt, C. Schulze-Briese, M. Suzuki, T. Tomizaki, H. Toyokawa and A. Wagner, J. Synchrotron Rad. 13: 120-130 (2006). 
[32] K. Ta Phuoc, S. Corde, C. Thaury, V. Malka, A. Tafzi, J. P. Goddet, R. C. Shah, S. Sebban and A. Rousse, Nature Photonics, 6, 308 (2012).

[33] M. Sanchez del Rio and R. J. Dejus, American Institute of Physics, Conference Proceedings, 705: 784 (2004).

[34] S. I. Salem and P. L. Lee, At. Data Nucl Data Tables, 18: 233-241(1976).

[35] http://wwwastro.mfsc.nasa.gov/xraycal/linewidths.html

[36] B. G. Gokhale, S. N. Shuka, and R. N. Srivastava, Phys. Rev. A, 28(2): 858-862 (1983).

[37] S. H. Glenzer, G. Gregori, F. J. Rogers, D. H. Froula, S. W. Pollaine, R. S. Wallace and O. L. Landen, Phys. Plasmas, 10(6): 2433-2441(2003).

[38] S. H. Glenzer, O. L. Landen, P. Neumayer, R. W. Lee, K. Widmann, S. W. Pollaine, R. J. Wallace, G. Gregori, A. Höll, T. Bornath, R. Thiele, V. Schwarz, W. D. Kraeft, and R. Redmer, Phys. Rev. Lett. 98: 065002(2007).

[39] M. K. Urry, G. Gregori, O. L. Landen, A. Pak and S. H. Glenzer, J. Quant. Spectrosc. Radiat. Transfer 99: 636-648(2006).

[40] R. S. Romaniuk, LCLS - Large Laser Infrastructure Development and Local Implications, Intl Journal of Electronics and Telecommunications, 60: 187(2014) 


\section{Figures}

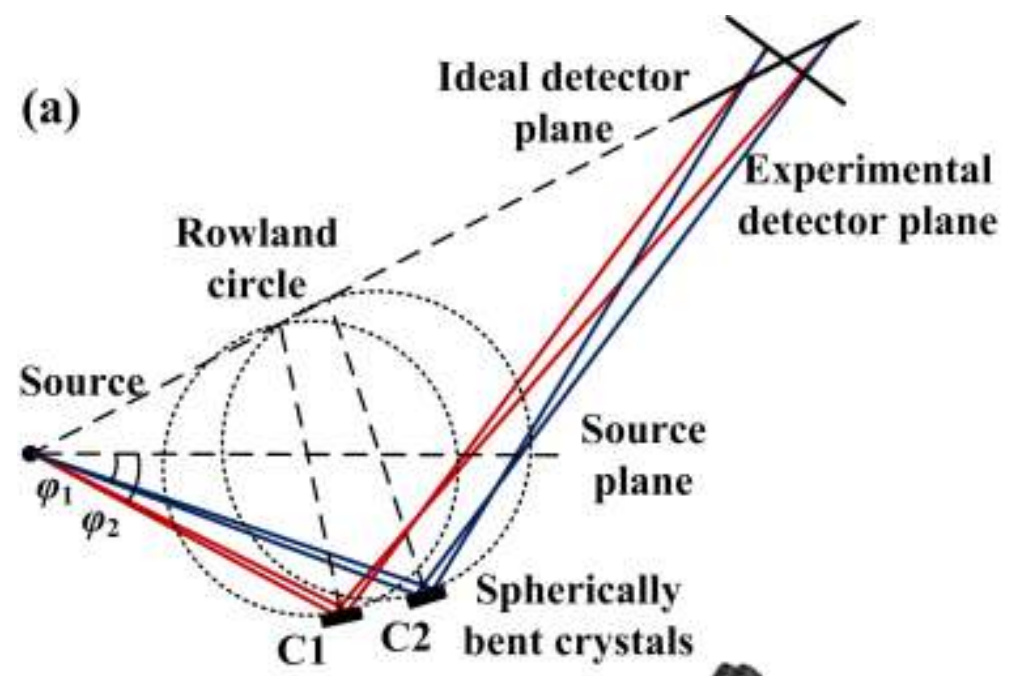

(b)

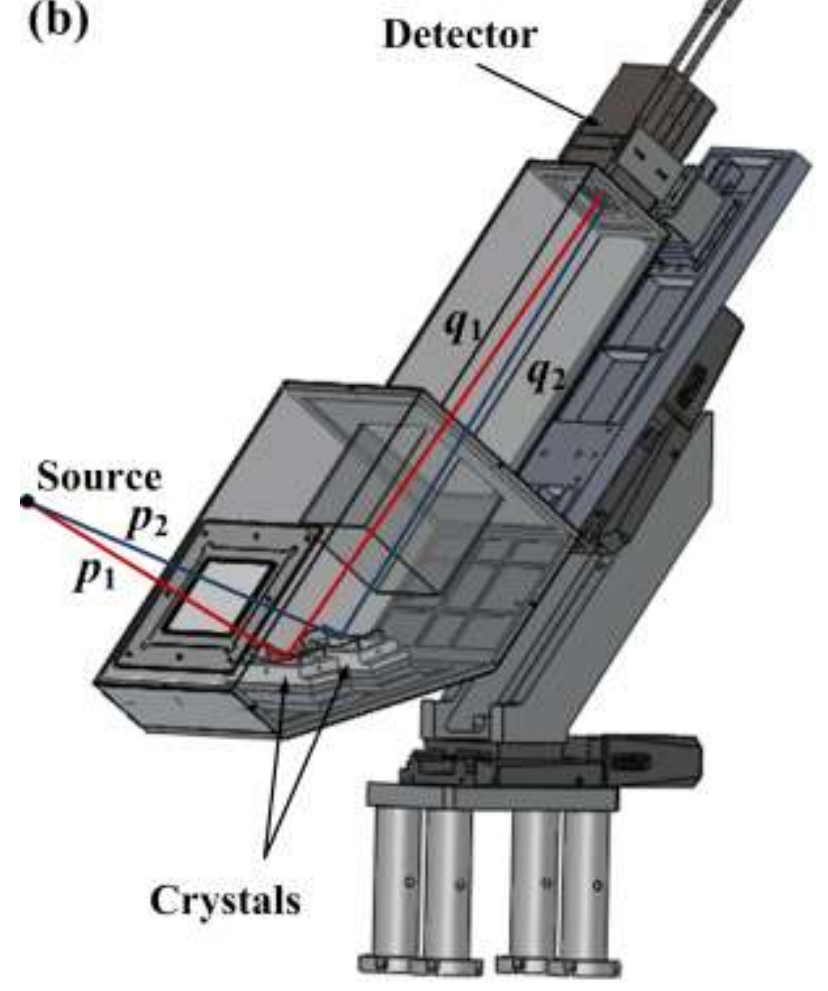

FIG. 1. (a) Schematic geometry of dual-channel x-ray imaging crystal spectrometer. The right crystal was tilted $\sim 0.5^{\circ}$ in the direction perpendicular with the paper plane with respect to the left one in the sagittal plane in order to separate the two spectra on a single detector. (b) CAD model of the XICS. Three linear translation stages were used to optimize the position of the detector for focusing and the spectrometer relative to the source. 
(a)

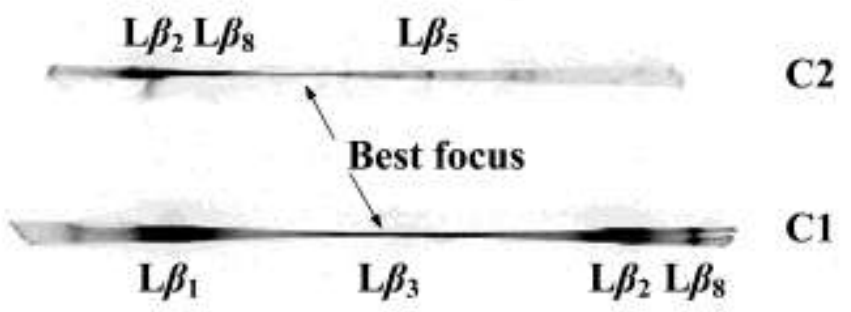

(b)

Ti K $\beta_{1} \quad \mathrm{~V} \mathrm{~K} \alpha_{2} \quad \mathrm{~V} \mathrm{~K} \alpha_{1}$

\section{C1}

Fig. 2. (a) Raw data obtained from a tungsten x-ray tube for calibration. The upper spectrum was recorded by $\mathrm{C} 2$ and the lower one was from $\mathrm{C} 1$ for different energy ranges . (b) Raw spectra data from Ti and $\mathrm{V}$ plasmas. 

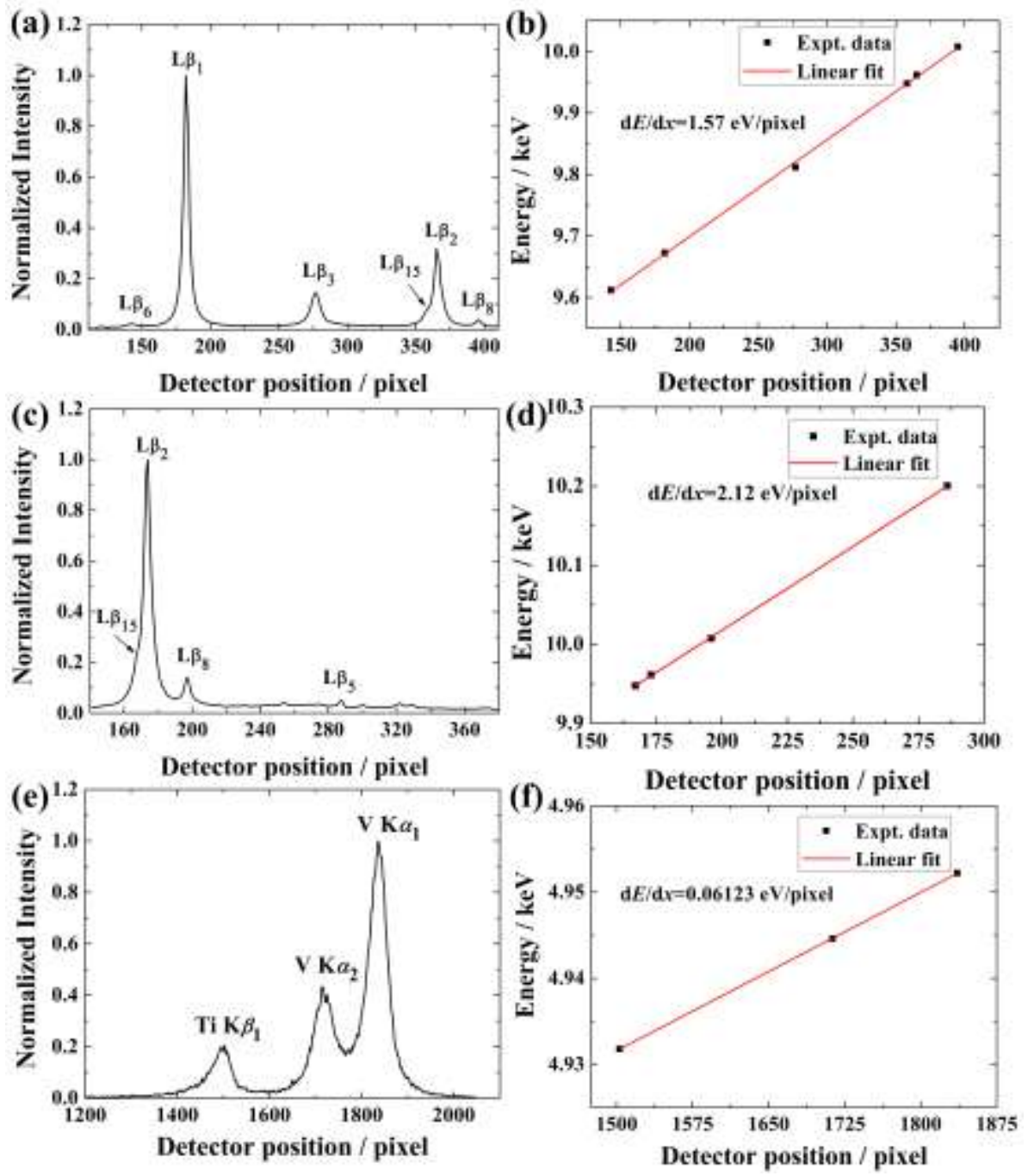

Fig. 3. Spectra from tungsten tube obtained by (a) $\mathrm{C} 1$ for the lower energy range and (c) (C2)

for the higher energy range. Linear fittings of the experimental data show constant linear

dispersions for (b) C1 and (d) C2. Both spectra were recorded in 2nd order reflection. (e) Spectra data of Ti and $\mathrm{V}$ radiation which was recorded by $\mathrm{C} 1$ in 1 st order reflection and (f) its linear fitting.

The main features are labeled. 

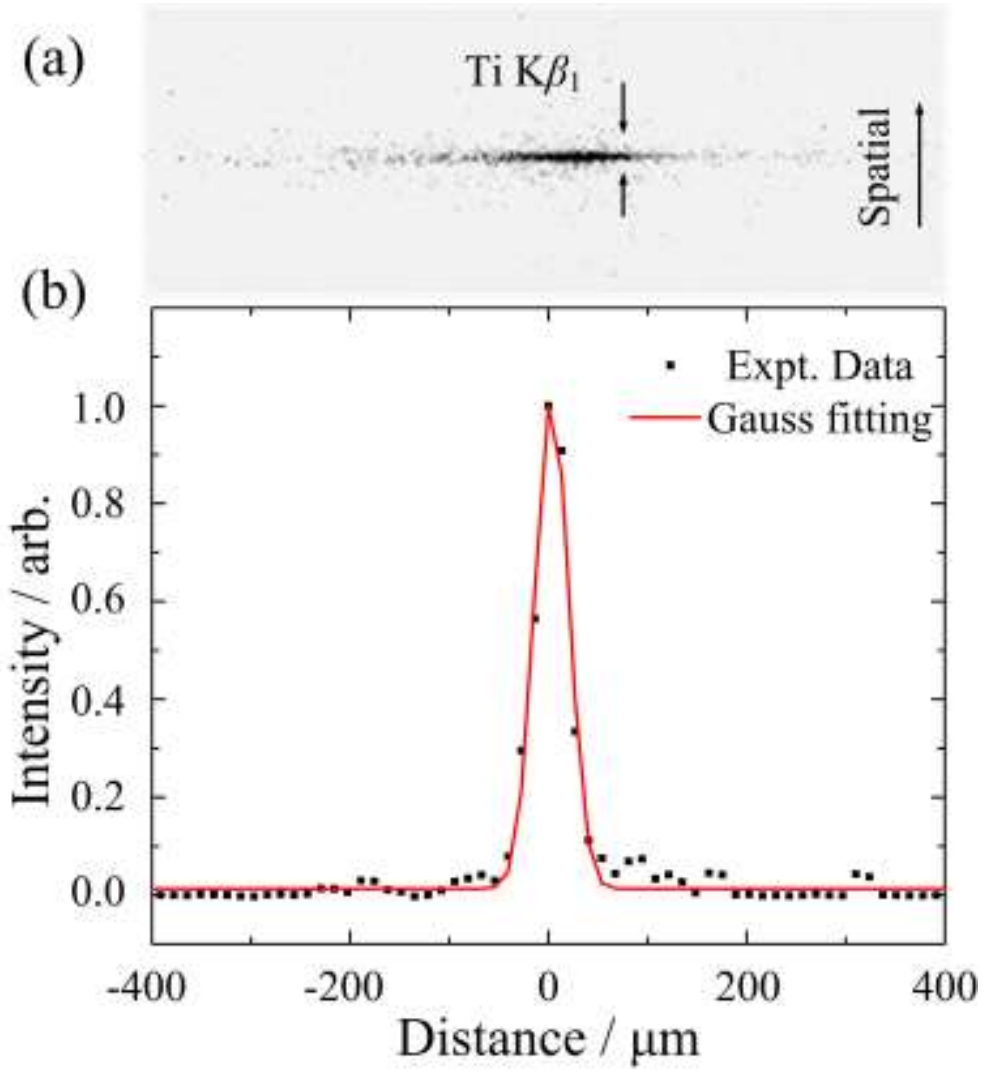

Fig. 4. (a) The raw data recorded by crystal 1 for $\mathrm{Ti} \mathrm{K} \beta_{1}$ line. (b) Spatial distribution of the $\mathrm{Ti}$ $\mathrm{K} \beta_{1}$ line in vertical direction. Gaussian fitting gives a FWHM of $\sim 40 \mu \mathrm{m}$ at the detector and 19.2 $\mu \mathrm{m}$ at the source.

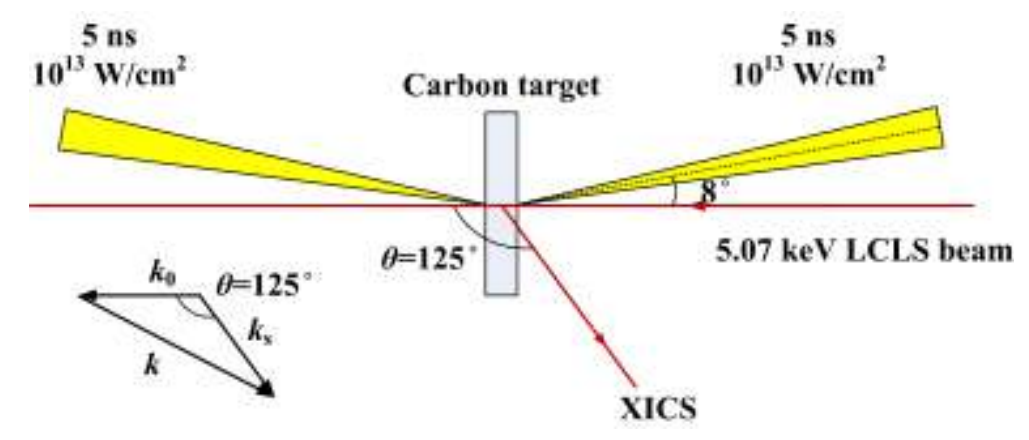

Fig. 5. Schematic of the scattering experiment used in MEC. Two heater laser beams compressed the carbon sample which was then probed by delayed x-ray beam from the LCLS XFEL at $5.07 \mathrm{keV}$. X-ray scattering spectra were observed in the downward direction with a scattering angle of $\sim 125^{\circ}$ using the crystal spectrometer. 
(a)

C2

C1

(b)

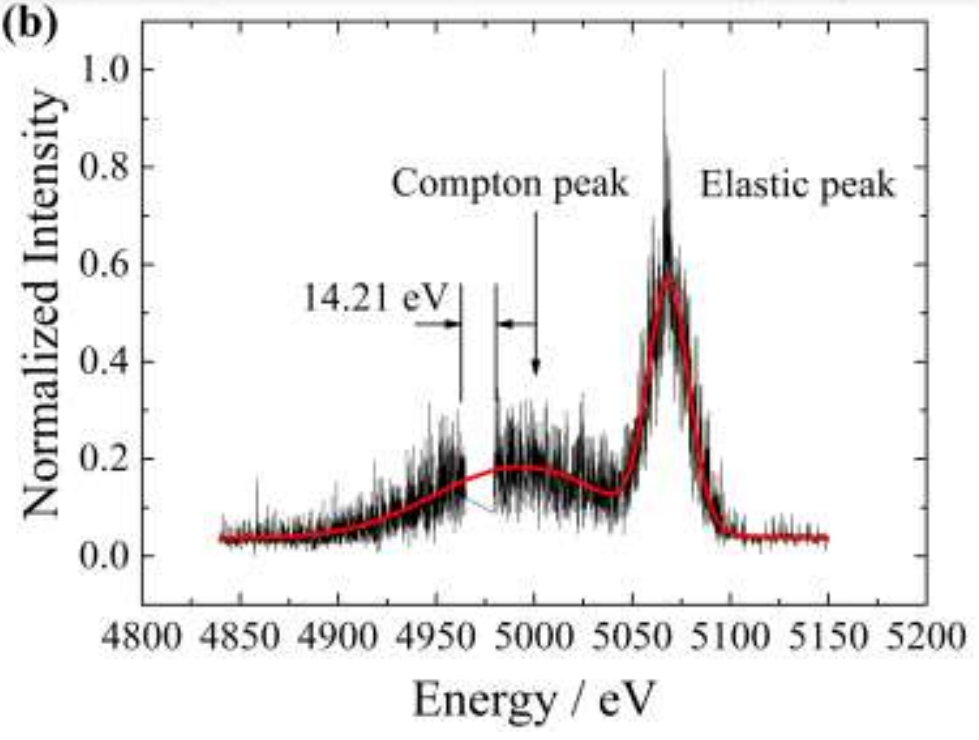

Fig. 6. (a) X-ray scattering raw data for $125^{\circ}$ scattering along with (b) fitted spectra. A 14.21 $\mathrm{eV}$ energy gap due to non-perfect alignment of the spectrometer is also seen. 

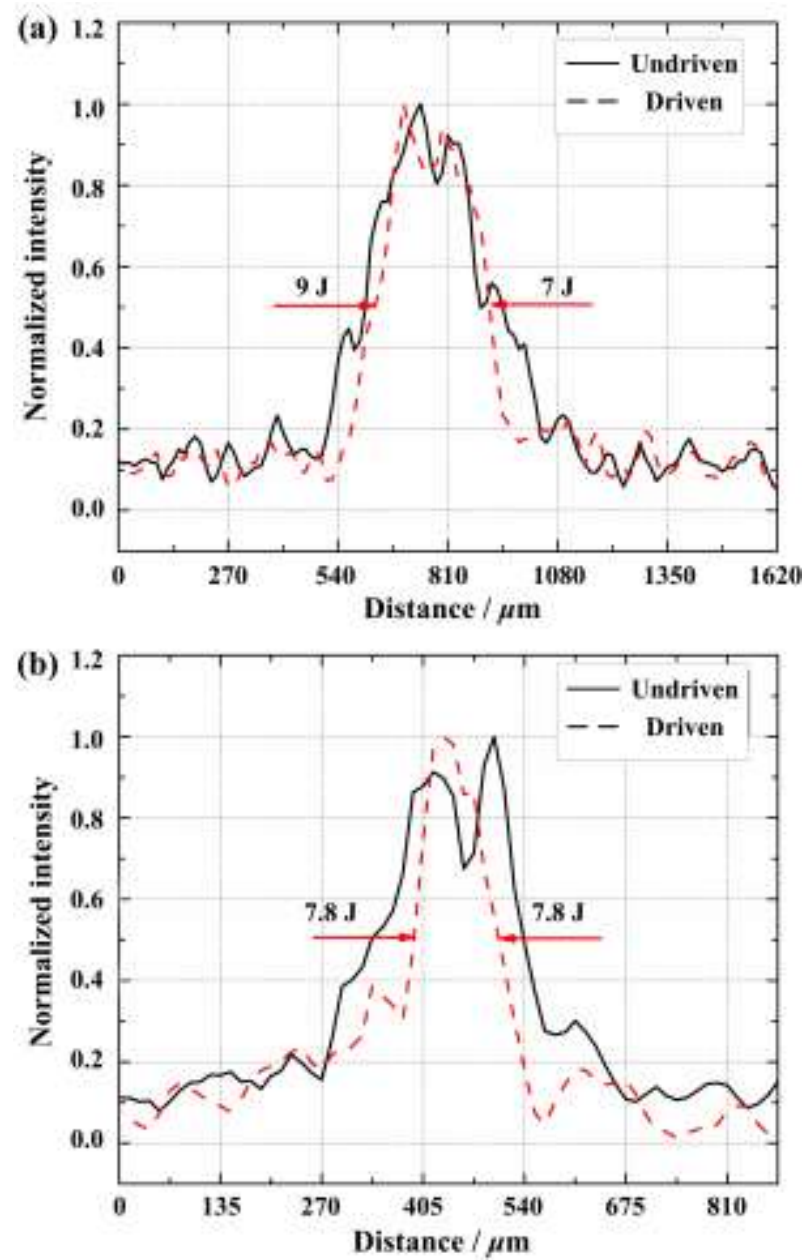

Fig. 7. Comparison of the spatial width of the elastic peak without driven lasers(solid line) and with driven lasers (dashed line) using (a) different laser energies and (b) the same laser energies. 